\title{
HACIA UN CATÁLOGO ACTUALIZADO DE LAS ORCHIDACEAE DE CUBA
}

\author{
ERnesto Múuica Benítez \\ Jardin Botánico Orquideario Soroa, Universidad de Pinar del Río \\ Apdo. Postal No. 5. Candelaria, Pinar del Río.Cuba. emujica@vrect.upr.edu.cu
}

Desde el siglo pasado las especies de orquídeas que habitan el territorio cubano han estado en el punto de mira de los estudiosos del tema por ser la isla de Cuba la mayor de las Antillas. Sin embargo, es hasta el año 1938 cuando se publica el primer estudio sobre las orquídeas de esta isla por parte de Julián Acuña Galé, prestigioso botánico cubano (Acuña Galé 1938). Posteriormente, en 1946, el Hermano León publica en su libro "Flora de Cuba" un nuevo tratamiento y listado (Sauget 1946). Un largo período de tiempo siguió a esta publicación y sólo 38 años después, en 1984, la Dra. Helga Dietrich, de la Universidad de Jena en Alemania, publica un checklist de acuerdo a los estudios realizados por ella en Cuba (Dietrich 1984). A fines del año 1998, después de un intenso trabajo, fue confeccionado por un grupo de autores del Orquideario Soroa, Cuba, el último listado oficial de géneros y especies de orquídeas registradas en Cuba, el cual salió publicado a principios del año 2000 formando parte del libro "Los Géneros de Orquídeas Cubanas" (Mújica et al. 2000).

Debido al dinamismo que tienen los estudios orquideológicos en el mundo y principalmente en Mesoamérica, son muchos los cambios nomenclatoriales y segregaciones que se han realizado después de tres años, por lo que ya se hace necesaria una nueva revisión de la familia para Cuba.

El objetivo del presente trabajo es la creación de un catálogo actualizado, teniendo en cuenta los nuevos registros, tendencias y resultados de los estudios taxonómicos actuales, llevados a cabo por importantes especialistas nacionales y extranjeros que durante años han dedicado sus esfuerzos a esta tarea.

Para ello se revisaron en su totalidad los ejemplares depositados en el Herbario del Instituto Superior Pedagógico de Pinar del Río (HPPR) (Mújica 2003), los del Herbario Johannes Bisse (HAJB), del Jardín Botánico Nacional y los depositados en el Herbario del Instituto de Ecología y Sistemática (HAC), que contiene las colecciones de los antiguos Herbario Sauvalle, Herbario de la Estación Agronómica de Santiago de Las Vegas y los del Herbario del Colegio de La Salle. Igualmente se han tenido en cuenta los ejemplares depositados en el Herbario del Orquideario Soroa, aunque éste no está actualmente registrado.

En los casos de especies que se encuentran en Cuba y no tienen testigo en ningún herbario nacional se ha confiado en algunos autores y en informaciones obtenidas de algunos herbarios extranjeros que no han podido ser visitados. Sólo se han hecho excepciones en los casos de especies citadas que carecen de testigos en herbarios nacionales o extranjeros pero que han sido o están siendo cultivadas en estos momentos en el Orquideario Soroa. Éstas están debidamente comprobadas por el autor después de ver los ejemplares, fotos y dibujos de los mismos, y así se hace constar en el listado.

Como podrá comprobarse, se han utilizado también datos e informes que aparecen tanto en antiguas bibliografías como en las más actuales, tratándose de llegar en todo momento a criterios que correspondan lo más fielmente posible a las tendencias que hoy se observan en el campo de la orquideología en Mesoamérica. Evidentemente se ha tratado de adoptar la nomenclatura más reciente en la designación de los taxa. Como todos saben, los estudios de análisis molecular han traído como consecuencia en algunos grupos la segregación de muchas de sus especies hacia otros géneros. En algunos casos estos cambios ya han sido aceptados en el listado en preparación, mientras que en otros el autor prefiere señalar estos nuevos cambios como sinónimos en espera de que los estudios con cada grupo concluyan, principalmente en los grandes, y poder ofrecer una realidad más completa de cada uno de ellos en el futuro. 
Igualmente se realizan una serie de comentarios en aquellos casos de cambios nomenclatoriales, adiciones o eliminaciones de géneros y especies registradas para el país y se señalan las especies endémicas en Cuba. El autor, en algunos casos, considera probable la presencia en territorio cubano de otras especies que actualmente carecen de un registro confiable, por lo que aparecen en un listado de especies de posible presencia en Cuba. Igualmente se brinda un pequeño listado de especies que han sido observadas en algunas ocasiones y sobre las cuales el autor muestra serias dudas.

Por último, el autor desea con el presente trabajo, lograr que sirva de referencia actualizada para aquellos que día a día se esfuerzan por estudiar y conservar las especies de esta familia de plantas en el país y la región.

\section{Agradecimientos}

Son muchas las personas que deben ser mencionadas y muy poco el espacio. En primer lugar al amigo Franco Pupulin del Jardín Botánico Lankester y principal gestor de esta idea junto a Carlos Ossenbach y su esposa Pilar Casasa, quienes desde San José de Costa Rica y al frente de la Fundación Lankester han colaborado desinteresadamente para la divulgación de este trabajo. A Eric Hágsater,
Helga Dietrich, León Ibarra, Robert Dressler, Carlyle A. Luer y James Ackerman por la paciencia mostrada y la ayuda brindada a la hora de responder a mis dudas. A los colegas del Orquideario Soroa, Rolando Pérez Márquez y José L. Bocourt y los técnicos por su apoyo en todo momento, a Alelí Morales, Curadora de la colección de la sección Orchidaceae del HAJB, al Dr. Armando Urquiola y Teresa García del HPPR y Juan Llamacho del HAC por las facilidades otorgadas para la revisión de esos herbarios.

\section{Literatura CitAdA}

Acuña Galé, J. 1938. Catálogo descriptivo de las orquídeas cubanas. Bol. Est. Agron. Santiago de Las Vegas 60.

Dietrich, H. 1984. Vorläufiges Gattungs- und ArtenVerzeichnis Kubanischer Orchidaceae. Wiss. Zeitschr. Friedrich-Schiller-Univ. Jena.

Mújica Benítez. E., 2003. Notas acerca de la colección de Orchidaceae del Herbario del Instituto Superior Pedagógico de Pinar del Río (HPPR), Cuba. Lankesteriana 6: 9-16.

Mújica Benítez. E., R. Pérez Márquez, J. Lazaro Bocourt Vigil, P.J. López Trabanco y T.M. Ramos Calderón. 2000. Géneros de Orquídeas Cubanas. Editorial Félix Varela, La Habana. 208 p.

Sauget, J.S. (Hno. León). 1946. Familia 2. Orquídeas. In Flora de Cuba. Vol. I. Gimnospermas. Monocotiledóneas. Contr. Occ. Mus. Hist. Nat. Col. La Salle 1 (8): 341-404.

Ernesto Mújica Benítez es Licenciado en Educación, convertido en estudioso de las orquídeas de forma autodidacta. Labora en el Orquideario Soroa desde hace 10 años, donde es Jefe de Colecciones y se dedica principalmente a los estudios fenológicos de las especies de orquídeas cubanas, además de incursionar en otras áreas de investigación. Ha participado e impartido conferencias y charlas sobre diversos temas en Argentina y Chile, además de haber visitado también Paraguay, Brasil y Costa Rica en funciones de trabajo. Actualmente está realizando sus estudios de doctorado sobre el estado de conservación de especies endémicas de la península de Guanahacabibes, de acuerdo a los resultados de los niveles de producción de frutos de las mismas. 